\title{
Therapeutic Effect of Alprostadil in Diabetic Nephropathy: Possible Roles of Angiopoietin-2 and IL-18
}

\author{
Changqing Luo ${ }^{\mathrm{a}}$ Ting $\mathrm{Li}^{\mathrm{a}}$ Chun Zhang ${ }^{\mathrm{a}}$ Qing Chen ${ }^{\mathrm{b}}$ Zhenqiong Lia Jianshe Liu ${ }^{\mathrm{a}}$ \\ Yumei Wang ${ }^{\text {a }}$ \\ aDepartment of Nephrology, Union Hospital, Tongji Medical College, Huazhong University of Science \\ and Technology, bDepartment of Hepatobiliary Surgery, Union Hospital, Tongji Medical College, \\ Huazhong University of Science and Technology, Wuhan, Hubei, The People's Republic of China
}

\section{Key Words}

Diabetic Nephropathy • Alprostadil • Angiopoietin-2 • IL-18 • Glomerular endothelial cells

\begin{abstract}
Background/Aims: To investigate the role of angiopoietin-2 (Ang-2) and IL-18 in the pathogenesis of diabetic nephropathy (DN) and the molecular mechanisms through which alprostadil protects renal function. Methods: DN was induced by streptozotocin and intraperitoneal injection of alprostadil was given to diabetic mice. After 2, 4 and 8 weeks of alprostadil treatment, the mRNA and protein expression of kidney Ang-2 and IL-18 were detected by reverse transcription PCR, Western blot and immunohistochemistry analyses. Mouse glomerular endothelial cells (GEnCs) were cultured in high glucose and treated with alprostadil. After transfection with an Ang-2-pcDNA and Ang-2-siRNA, both Ang-2 and IL-18 expression were measured by Western blot analyses. Results: Alprostadil treatment caused a significant decrease in the renal damage parameters. Both Ang-2 and IL-18 were significantly increased in DN mice and in GEnCs cultured in high glucose; however, their expression was greatly reduced by alprostadil treatment. Ang-2 could also increase IL-18 expression in cultured endothelial cells under high glucose, and this response was partially blocked by Ang-2 siRNA. Conclusions: Ang-2 and IL-18 may be associated with the development and progression of DN in mice. Alprostadil treatment can protect renal function by reducing proteinuria. These effects are mediated, at least in part, through down-regulation of Ang-2 and IL-18 expression.
\end{abstract}

Copyright $(2014$ S. Karger AG, Basel

\section{Introduction}

Diabetic nephropathy (DN) is the most common cause of end-stage renal disease, and it is present in $25-40 \%$ of those entering into chronic dialysis care [1]. Risk factors for DN 
include hyperglycemia, hypertension, glomerular hyperfiltration, proteinuria, and possibly reduced nephron number and lipid disorders [2]. Despite clinical advances in understanding the metabolic causes of nephropathy, treatments aimed at interrupting these pathological processes have had limited success. Therefore, early intervention and treatment of DN is particularly important. However, the pathogenesis of DN is not yet fully understood, which has caused some difficulties in its diagnosis and subsequent treatment.

In recent years, the role of the angiogenesis-related factors of the angiopoietin family have received extensive attention in the context of DN $[3,4]$. Studies have found that members of the angiopoietin family and the vascular endothelial growth factor (VEGF) family may be involved in the development of DN $[3,5]$. Among the four structurally related proteins of the angiopoietin family (Ang-1, Ang-2, Ang-3 and Ang-4), Ang-2 is known to be the ligand of the Tie receptor, tyrosine kinase. In vitro experiments revealed that Ang- 2 expression can be stimulated by glucose levels [6]. In vivo experiments further showed that Ang-2 expression was significantly increased in the renal cortex in a type 1 DN model [7]. Alterations in Ang-1 and Ang-2 expression were also associated with uncontrolled changes in capillary growth and structural changes in DN [3]. Studies have also found that high levels of Ang-2 expression can induce apoptosis of endothelial cells and increase urinary protein [8]. However, its role in DN and the mechanisms by which it exerts its effects remain unclear.

Previous studies have suggested that chronic low-grade inflammation and activation of the innate immune system are closely related to the microvascular complications underlying diabetes [9]. Inflammatory cytokines such as IL-1, IL-6, IL-18 and TNF- $\alpha$ maybe involved in the development and progression of DN [9]. For example, accumulating evidence suggests that IL-18 is a DN pathogenic cytokine. It has been reported that serum and urine IL-18 increase in patients with type 2 diabetes, which may be associated with the occurrence of DN $[10,11]$. However, how IL-18 functions in the pathogenesis of DN has not been fully elucidated.

Alprostadil (PGE1) is widely used in the clinic based on its vasodilatory effects to dilate blood vessels and its anti-platelet effects to inhibit platelet aggregation. Clinical studies also show that alprostadil can reduce urinary protein and protect renal function through unclear mechanisms [12-14]. In this study, we sought to establish a DN model by STZ induction, as a means to observe the early morphological changes that occur in DN, while simultaneously monitoring the expression levels of key angiogenesis-related factors such as, Ang-2/Tie2 and the inflammatory cytokine, IL-18. Our data provide new insights into the molecular mechanism by which alprostadil reduces urinary protein and protects renal function, that is, at least in part due to a decrease in Ang-2 and IL-18 levels.

\section{Materials and Methods}

Induction of diabetic nephropathy in mice

All animal protocols were performed in accordance with the U.S. National Institutes of Health guidelines (NIH Publication No.85-23, revised 1996) and approved by the Animal Care and Use Committee at the Huazhong University of Science and Technology.

The diabetic mouse model was induced as described previously [15-17]. Briefly, 8-week-old male C57BL/6J mice at SPF level (purchased from Beijing HFK Bioscience Co., Ltd., Beijing, China) were divided randomly into three groups: non-diabetic controls $(n=15)$, diabetic nephropathy model $(n=20)$ and diabetic mice with alprostadil treatment $(n=25)$. Mice were fasted for $>12 \mathrm{~h}$ and injected intraperitoneally with a single dose of $140 \mathrm{mg} / \mathrm{kg}$ of STZ (streptozotocin; Sigma-Aldrich, St. Louis, MO, USA) solubilized in pH4.8 citrate buffer or saline. Three days after administration, blood was withdrawn from the tail vein and blood glucose was measured using a glucometer (One Touch Blood Glucose Instrument, LifeScan, USA). Mice were considered diabetic if blood glucose was higher than $16.7 \mathrm{mmol} / \mathrm{L}$ for two consecutive measurements [15, 18]. $\mathrm{HbA}_{1 \mathrm{c}}$ levels were measured using a commercial kit ( $\mathrm{A} 1 \mathrm{C}$ now ${ }^{+}$; Bayer Corporation, NY, USA). Urinary protein was monitored weekly and mice with continuous proteinuria were confirmed as having DN. Body weights were measured daily throughout the study. 
After two weeks of STZ injection, mice were considered diabetic. Based on our previous studies [19] and unpublished pilot experiments, diabetic mice were given daily intraperitoneal injections of alprostadil (Beijing Tide Pharmaceutical Co., Ltd., Beijing, China) at a dose of $80 \mu \mathrm{g} / \mathrm{kg}$ or saline as a control. After two weeks, four weeks and eight weeks of drug treatment, urine samples were collected from each group using metabolic cages [17]. At the end of the study, 15 control mice, 15 diabetic mice and 18 diabetic mice treated with alprostadil were sacrificed by cervical dislocation and blood samples were collected. Kidney weight was measured immediately. Subsequently, half of the middle portion of the left kidney was fixed in $4 \%$ paraformaldehyde for detection of renal morphology and immunohistochemical staining while the rest of the kidney was snap frozen at $-80^{\circ} \mathrm{C}$ for RT-PCR and Western blot analysis.

\section{Urinary protein excretion, blood lipids, and renal function determination}

Urinary protein, triglycerides (TG), total cholesterol (TC), serum creatinine and urinary creatinine were determined at 24h using an automatic biochemical analyzer (ADVIA 2400, Siemens, Erlangen, Germany) as previously described [16]. Creatinine clearance rate was calculated and expressed as $\mu \mathrm{L} / \mathrm{min} / \mathrm{g}$.

Renal morphology assessment

Mouse kidney tissue was fixed in 4\% paraformaldehyde and embedded in paraffin. Tissues were serially sectioned at a thickness of $4 \mu \mathrm{m}$, dewaxed with xylene, hydrated with gradient ethanol before staining with PAS. Renal morphological changes were observed under a light microscope. As previously described [16], PAS-stained sections of the renal cortex, the glomerular tuft area was determined by the total glomerular area minus the urinary space area and the urinary recesses area. The ratio of the glomerular matrix area to tuft area was taken as the relative glomerular extracellular matrix (ECM). At least 50 glomeruli were counted per kidney and the average values determined for these glomeruli were used for quantitative analysis. Quantitative evaluation was performed using an Olympus light microscope equipped with a video camera and an HPIAS-1000 image analysis system at 400x magnification [20].

\section{Cell culture and treatment}

Mice glomerular endothelial cells (GEnCs) were purchased from Cell Biologics (C57-6014G) and cultured according to the manufacturer's protocol and previous reports $[21,22]$. Cells were grown in Dulbecco's modified Eagle's medium (DMEM) (Gibco Invitrogen, Life Technologies, Scotland, UK) with 10\% fetal bovine serum (FBS, Gibro Invitrogen). Cells were digested with $0.25 \%$ Trypsin-EDTA solution (Gibro Invitrogen) for $3-5 \mathrm{~min}$ at $37^{\circ} \mathrm{C}$. As soon as the cells detached, cell culture medium supplemented with $10 \%$ FBS were added to these cells. Then cells were plated in six-well plates coated with gelatin-based coating solution in a humidified, $5 \% \mathrm{CO} 2 /$ incubator at $37^{\circ} \mathrm{C}$. GEnCs were grown to confluence at $2 \times 10^{4}$ cells $/ \mathrm{ml}$, cells were then incubated in DMEM without FBS for 24 hour. After incubation with 5.6 mM D-glucose (as control, NG group) and $30 \mathrm{mM}$ D-glucose (HG group) for 12, 24 or 48 hours, the culture supernatants were collected and the levels of Ang-2 and IL-18 were determined by Western blot analysis.

\section{Ang2 transfection studies in GEnCs}

Once the GEnC cells reached $80-90 \%$ confluence $\left(5 \times 10^{4} \mathrm{cell} / \mathrm{ml}\right)$, the medium was replaced with OPTIMEM (Invitrogen, Carlsbad, CA, USA) for 4 hours and incubated with $5 \% \mathrm{CO}_{2}$ at $37^{\circ} \mathrm{C}$. Subsequently, the pcDNA3.1-Ang2 plasmid (kindly provided by Dr. Benest, University of Heidelberg), Ang2 siRNA and Lipofectamine 2000 diluted with OPTI-MEM were applied to the cells with the six-well plates gently rattled for 6 hours. Ang2 siRNA (Santa Cruz Biotechnology) using Lipofectamine 2000 (Invitrogen, USA) according to manufacturer's protocols in the absence and presence of various concentrations of glucose. The OPTI-MEM media was replaced with normal medium and incubated for another 48 hours. Cell culture supernatants were collected and Ang-2 as well as IL-18 levels were measured using Western blot analysis.

Reverse transcription PCR (RT-PCR) assay

Total RNA was extracted from renal tissues using Trizol reagent (Takara, Shiga, Japan) according to manufacturer's instructions. Three $\mu$ g of total RNA was used to synthesize cDNA with Prime Script ${ }^{\mathrm{TM}}$ synthesis kit (Takara). PCR amplification was performed in the presence of $2 \mu \mathrm{l}$ cDNA, TaqDNA Polymerase (Takara) and gene specific primers. The following primers were used: Ang-2, sense (5'-ATCTTCCTCCAGCCCCTAC AT$3^{\prime}$ ) and antisense (5'-GCTTCCACATCAGTCAGTTTCC-3); Il-18, sense (5'-GACCTGGAATCAGACAACTTTGG-3) 
and antisense( $5^{\prime}$-GCCTCGGGTATTCTGTTATG GA-3'); Ang-1, sense (5'-ATCTTCCTCCAGCCCCTACAT-3) and antisense (5'-TGTTTGATTTAGTACCTGGGTCTCA-3); VEGF, sense(5'-AGGCAGACTATTCAGCGG ACTC-3) and antisense (5'-CTCTTTCGTCTGCTGATTTCCAC-3); Tie 2, sense (5'-AACCTGTTCACCTCAGCCTTCA-3) and antisense (5'-CTGGTCCACTACACCTT TCT TTAC-3); $\beta$-actin, sense (5'-CTGTCCCTGTATGCCTCTGGTC-3) and antisense (5'-CTCTTTGATGTCACGCACGATTTC-3). PCR products were separated by $2 \%$ agarose gel electrophoresis $(2 \mu$ l Syber Green I was added to each $8 \mu$ l PCR product). Results were obtained with an automatic gel imaging system (Media Cybernetics, MD, USA) and DNA band intensity was quantified using Gel-Pro Analyzer 4.0 software (Media Cybernetics). $\beta$-actin was used as an internal control and data are shown as relative gene expression levels normalized to $\beta$-actin.

\section{Western blot analysis}

Western blot was performed as described previously [23]. Briefly, after protein concentrations were determined (BCA Protein Assay Kit, Sigma-Aldrich), $50 \mu \mathrm{g}$ protein lysates were separated via a 10\% SDSPAGE and transferred onto polyvinylidene difluoride membranes (Sigma-Aldrich, USA). Non-specific binding was blocked by incubating membranes in 5\% fat-free milk in TBS buffer containing $0.1 \%$ Tween 20 (TBS-T) for 1 hour. Thereafter, membranes were incubated overnight at $4{ }^{\circ} \mathrm{C}$ with primary antibodies for goat anti-angiopoietin-2 (1:1000, Santa Cruz Biotechnology, CA, USA) and rabbit anti-IL-18 (1:1000, Santa Cruz Biotechnology). After washing, blots were incubated for 1 hour at room temperature with horseradish peroxidase (HRP) labeled secondary antibodies (1:5000). Immunoreactive proteins were developed by enhanced chemiluminescence (ECL) Western blotting detection reagent (Amersham Biosciences, Piscataway, NJ, USA) and visualized on Kodak Omat X-ray films. $\beta$-actin was used as a loading control. The density of each band was quantified using Image J software (NIH, Bethesda, MD, USA).

\section{Immunohistochemistry}

Four micron paraffin sections were hydrated through a graded series of ethanol washes, followed by $3 \% \mathrm{H}_{2} \mathrm{O}_{2}$ treatment, microwave heating ( $2 \times 3 \mathrm{~min}$ at high power), and three PBS washes. Sections were then blocked in 5\% BSA for 20min and incubated with rabbit anti-mouse Ang-2 antibody (Santa Cruz Biotechnology, 1:150) or rabbit anti-mouse IL-18 antibody (Santa Cruz Biotechnology, 1:150) at $4{ }^{\circ} \mathrm{C}$ overnight. After PBS washes, slices were incubated with a HRP-conjugated secondary antibody at $37^{\circ} \mathrm{C}$ for 30 min before staining with $\mathrm{DAB}$ and hematoxylin. Semi-quantitative analysis of target protein expression was performed as previously described3 [24]. Briefly, ten randomly selected fields (400x) per kidney were captured and each field was imaged with one glomerulus in the center of the picture. A positive area was identified by yellow staining and Image-Pro Plus software (Media Cybernetics, MD, USA) was used to quantify the integrated optical density (IOD). The ratio of positive area to total area represents the relative amount of substance expression.

\section{Statistics}

Data are expressed as mean \pm SEM. Statistical significance was calculated using one-way ANOVA, followed by post hoc analysis using the least significant difference method. Bi-variate correlation analysis was performed using Pearson correlation analysis. Values were considered statistically significant when $\mathrm{P}<0.05$.

\section{Results}

\section{Alprostadil treatment had no effect on metabolic parameters in mice}

STZ-injected mice displayed increased blood glucose and HbA1c levels as well as decreased body weight prior to alprostadil treatment, compared with non-diabetic control mice (Fig. 1). Alprostadil was shown to have no obvious effect on these metabolic parameters throughout the treatment period (Fig. 1). There were also no significant differences in serum lipid levels among all three groups (data not shown).

Alprostadil treatment ameliorated renal damage

STZ-injected mice displayed an increased urinary protein excretion (diabetic: $1.26 \pm$ 0.13 versus non-diabetic: $0.27 \pm 0.03$ ) at $24 \mathrm{~h}$, indicative of renal damage and successful 
Fig. 1. Alprostadil has no effect on blood glucose, body weight and HbA1c levels. Blood glucose (a), body weight (b), HbA1c (c) were measured in non-diabetic control mice ( $\mathrm{N}$, open circles), diabetic mice (D, open triangles) and diabetic mice treated with alprostadil (A, black circles) for various time courses after injection of alprostadil. ( $n=5-6$ each group).

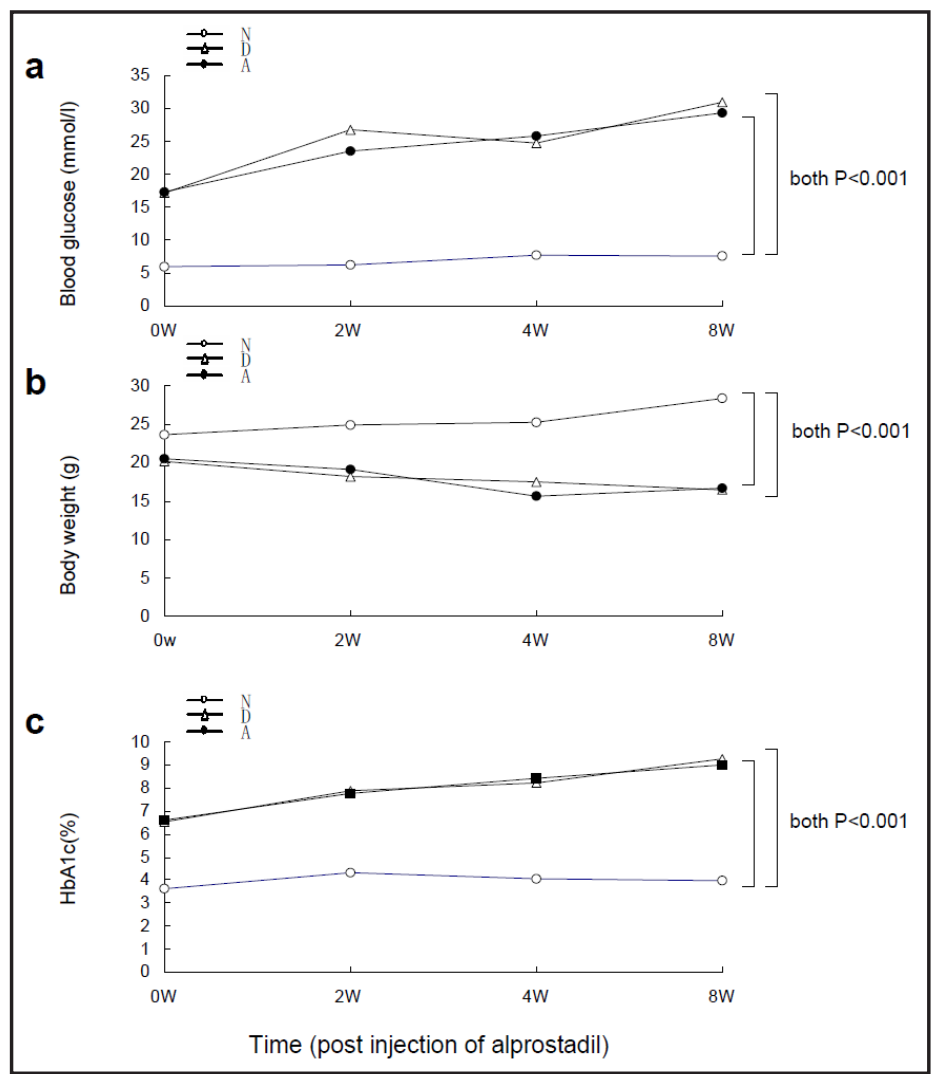

establishment of DN. Twenty-four hour urinary protein, creatine clearance rate and kidney/ body weight ratio were all significantly increased in diabetic mice compared with nondiabetic mice ( $\mathrm{P}<0.001$, Fig. 2). After four weeks of daily treatment with alprostadil $(80 \mu \mathrm{g} /$ kg BW intraperitoneal injection), the $24 \mathrm{~h}$ urine protein (Fig. 2a), creatinine clearance rate (Fig.2b) and kidney/body weight ratio (Fig. 2c) were significantly reduced compared with diabetic mice $(\mathrm{P}<0.05)$. Furthermore, with prolonged (eight weeks) alprostadil treatment, $24 \mathrm{~h}$ urinary protein and creatinine clearance rates were still reduced compared with diabetic mice (Fig. 2a and b).

\section{Alprostadil treatment ameliorated renal morphologic lesions}

We next examined pathological changes after alprostadil treatment by Periodic acidSchiff (PAS) staining. As shown in Fig. 3a, compared with non-diabetic control mice, diabetic mice showed glomerular hypertrophy, expansion of the mesangial area, and cloudy swelling of renal tubular epithelial cells. Moreover, the pathological damage increased over time (Fig. 3a, panels B1-B3). However, these pathological changes were ameliorated as short as two weeks after alprostadil treatment when compared to control diabetic mice, and continued to improve with prolonged treatment time (Fig. 3a, panels C1-C3). Furthermore, quantitative analysis showed that the ECM was significantly reduced after four weeks of alprostadil treatment in diabetic mice (Fig. 3 b).

Hyperglycemia-induced increase of Ang-2 levels was reduced after alprostadil treatment

Due to our previous observations of Ang2 levels increased in DN patients and others highlighting a potential role for Ang2 in DN [4, 6, 7], we examined the expression of Ang2 in STZ-induced and alprostadil-treated mouse models. Ang-2 mRNA levels were greatly increased in diabetic mice as compared to non-diabetic mice (Fig. 4a, b), consistent with previous reports ${ }^{7}$. Consistent with our RNA findings, Ang-2 protein levels were also greatly increased in diabetic mice (Fig. 4c-d). However, alprostadil treatment significantly reduced both Ang-2 mRNA and protein levels (Fig. 4a-d). Immunohistochemistry staining studies 


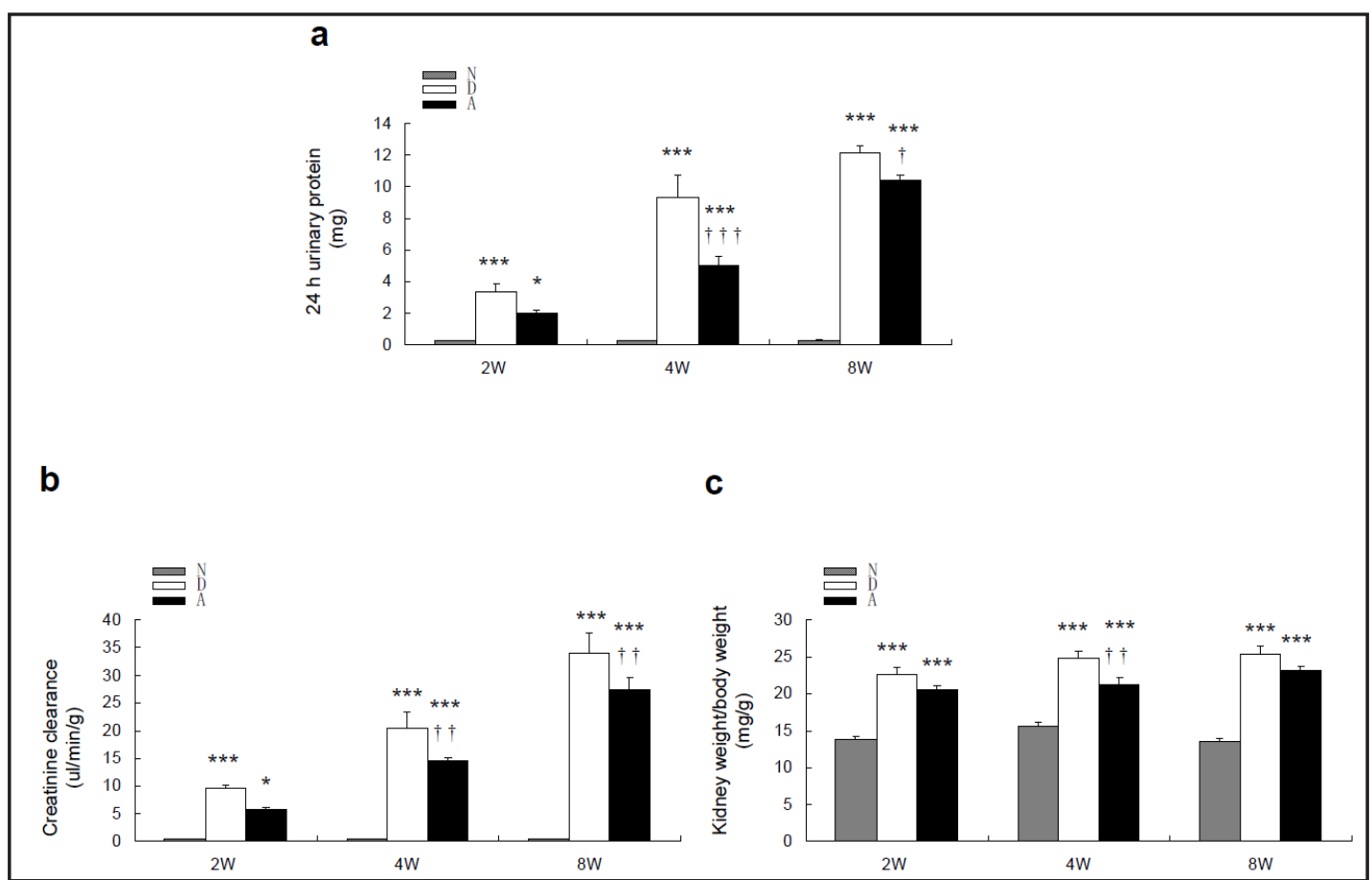

Fig. 2. Alprostadil treatment ameliorated renal damage. After four weeks of alprostadil treatment, (a) $24 \mathrm{~h}$ urinary protein, (b) creatinine clearence rate and (c) the ratio of kidney/body weight were ameliorated in diabetic mice. N: non-diabetic mice (hatched bar); D: diabetic mice (open bar); A: diabetic mice treated with alprostadil (black bar). ${ }^{*} \mathrm{P}<0.05$ vs. non-diabetic mice, ${ }^{* * *} \mathrm{P}<0.001$ vs. non-diabetic mice, $\uparrow \mathrm{P}<0.05$ vs. diabetic mice, $\uparrow \uparrow \mathrm{P}<0.01$ vs. diabetic mice, $\uparrow \uparrow \uparrow \mathrm{P}<0.001$ vs. diabetic mice. ( $\mathrm{n}=5-6$ each group).

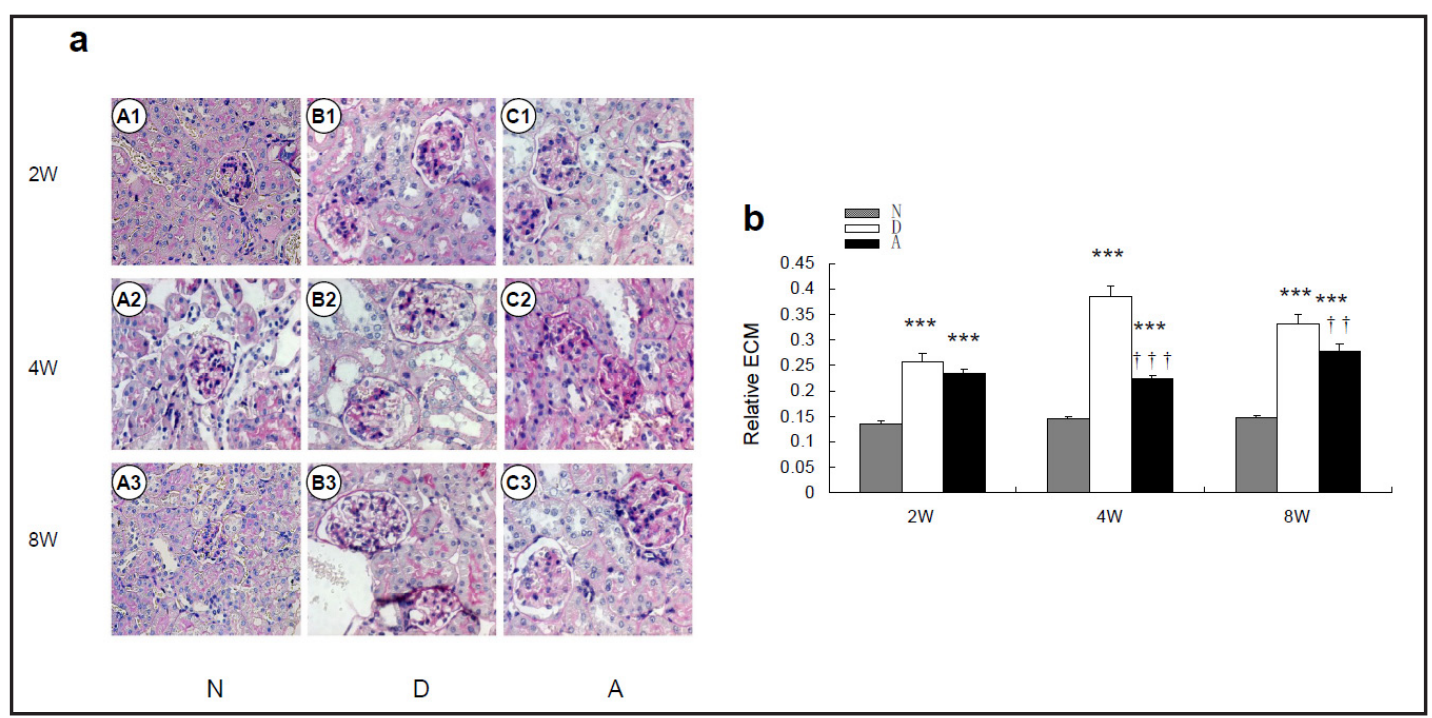

Fig. 3. Alprostadil treatment ameliorated renal lesions. (a) Representative light microscopic images of glomeruli stained with periodic acid-Schiff (400x). (b) Quantitative analysis of glomerular extracellular matrix (ECM) at various time courses. N: non-diabetic mice (hatched bar); D: diabetic mice (open bar); A: diabetic mice treated with alprostadil (black bar). ${ }^{* * *} \mathrm{P}<0.001$ vs. non-diabetic mice, $\uparrow+\mathrm{P}<0.01$ vs. diabetic mice, $+\uparrow+\mathrm{P}<0.001$ vs. diabetic mice. ( $\mathrm{n}=5-6$ each group).

revealed that Ang-2 was mainly expressed in glomeruli, alongside the capillary loops (arrowhead) and not detected in renal tubular epithelial cells (Fig. 4e). 
a

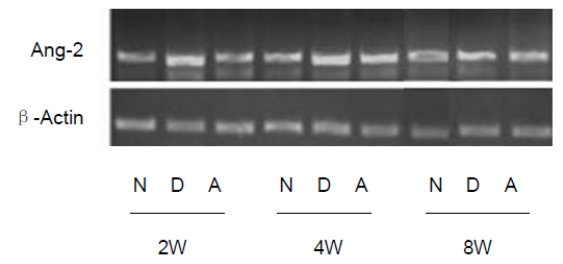

b

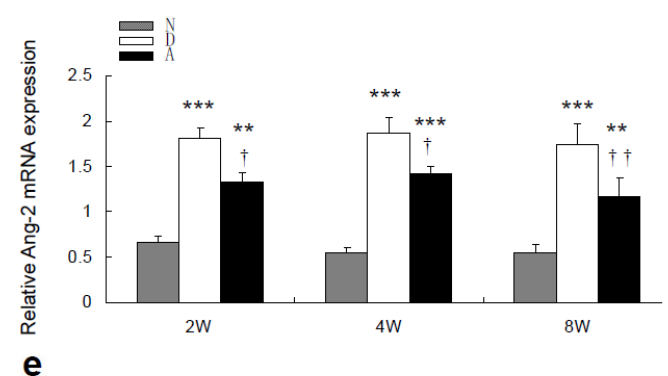

e

$2 \mathrm{~W}$

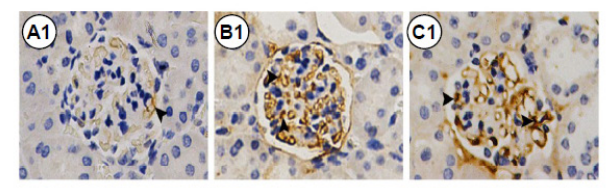

$4 \mathrm{~W}$

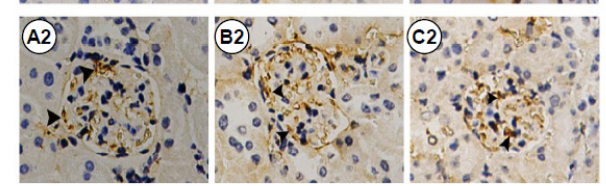

$8 \mathrm{~W}$

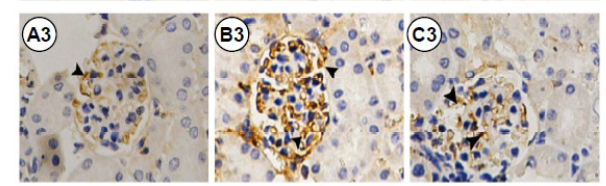

C
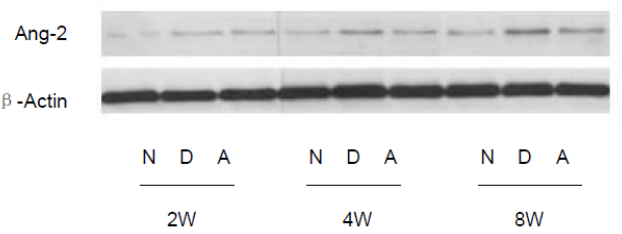

d

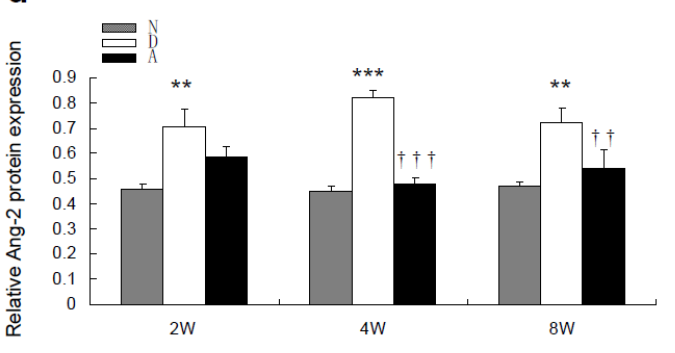

$\mathrm{N}$

D

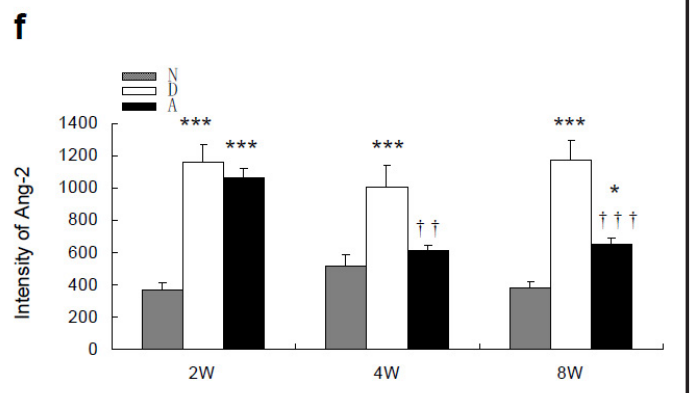

Fig. 4. Alprostadil suppresses angiopoietin-2 expression. (a, b) Ang-2 gene expression was assessed by semi-quantitative RT-PCR. (c, d) Protein levels were analysed by Western blot and (e, f) immunohistochemistry. Representative images of renal sections (e, 400x) show Ang-2 expressed alongside capillary loops (arrowhead), but no staining was detected in the tubular epithelial cells. N: non-diabetic mice (hatched bar); D: diabetic mice (open bar); A: diabetic mice treated with alprostadil (black bar), ${ }^{*} \mathrm{P}<0.05$ vs. non-diabetic mice, ${ }^{* *} \mathrm{P}<0.01$ vs. non-diabetic mice, ${ }^{* * *} \mathrm{P}<0.001$ vs. non-diabetic mice, $\uparrow \mathrm{P}<0.05$ vs. diabetic mice, $\uparrow \nmid \mathrm{P}<0.01$ vs. diabetic mice, $\uparrow+\uparrow P<0.001$ vs. diabetic mice. ( $\mathrm{n}=5-6$ each group).

We also examined expression levels of other angiopoietin family members such as Ang1 and Tie 2 as well as vascular endothelial growth factor (VEGF). No significant difference in mRNA expression was observed among all of the experimental groups (data not shown).

\section{Alprostadil treatment suppressed IL-18 expression in diabetic mice}

Similar to Ang-2, both mRNA and protein levels of IL-18 were upregulated in diabetic mice compared with non-diabetic group, consistent with previous reports $[10,11]$. Moreover, four weeks of alprostadil treatment significantly reduced both mRNA and protein levels of IL-18 in diabetic mice (Fig. 5a-d). We also performed immuno-histochemistry staining for IL-18 in kidney. As shown in Fig. 5e, IL-18 was expressed mainly in the glomeruli, alongside the capillary loops (arrow), with much lower expression in tubular epithelial cells. The 


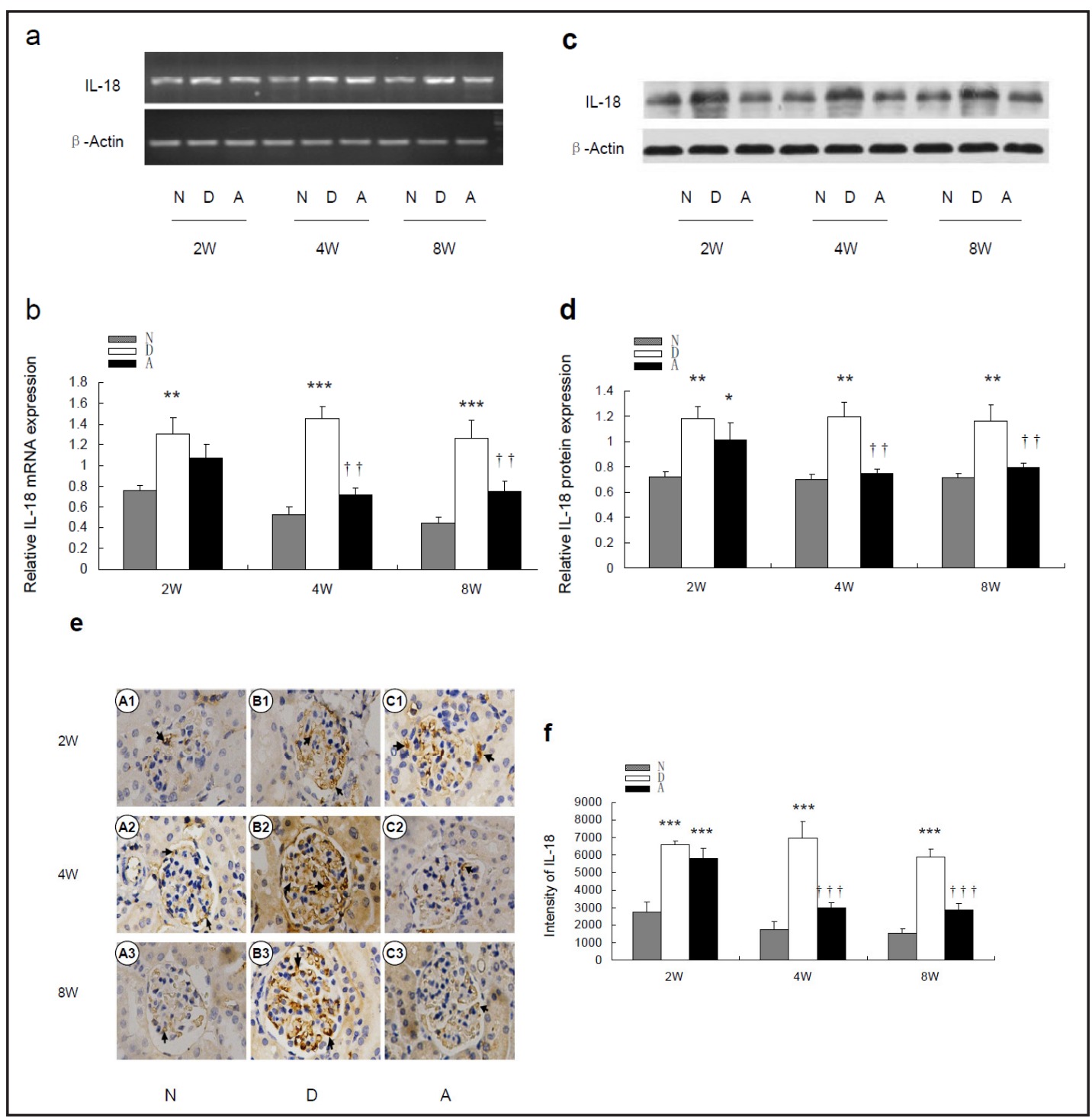

Fig. 5. Alprostadil suppresses IL-18 expression. (a, b) IL-18 expression was assessed by semi-quantitative RT-PCR. (c,d) Protein levels was analysed by Western blot and (e,f) immunohistochemistry. Representative images of renal sections (e, 400x) shows abundant IL-18 expressed in the glomerular alongside the capillary loops and a small quantity staining the tubular epithelial cells (arrow). N: non-diabetic mice (hatched bar); D: diabetic mice (open bar); A: diabetic mice treated with alprostadil (black bar), ${ }^{*} \mathrm{P}<0.05$ vs. non-diabetic mice, ${ }^{* *} \mathrm{P}<0.01$ vs. non-diabetic mice, ${ }^{* * *} \mathrm{P}<0.001$ vs. non-diabetic mice, $\dagger+\mathrm{P}<0.01$ vs. diabetic mice, $\dagger \dagger+\mathrm{P}$ $<0.001$ vs. diabetic mice. ( $n=5-6$ each group).

staining intensity of IL-18 was significantly greater in diabetic mice than in non-diabetic controls. Four weeks of alprostadil treatment dramatically reduced IL-18 levels in diabetic mice (Fig. 5e and f).

\section{Correlation of Ang-2 and IL-18 expression}

We also observed a positive correlation between Ang-2 and IL-18 mRNA levels (Fig.6a). Similarly, Ang-2 protein levels positively correlated with IL-18 protein levels (Fig. 6b and c), as analyzed by quantification of Western blot and immunohistochemistry data. Taken together, our data suggest that alprostadil may play a role in regulating Ang-2 and IL-18 expression. 
Fig. 6. Correlation analysis between Ang-2 and IL-18. (a) Ang-2 mRNA expression correlated positively with IL-18 mRNA expression. (b, c) Ang-2 protein levels correlated positively with IL-18 protein levels. (b: represents variables from Western blot quantification and c: represents variables from immunohistochemistry quantification).

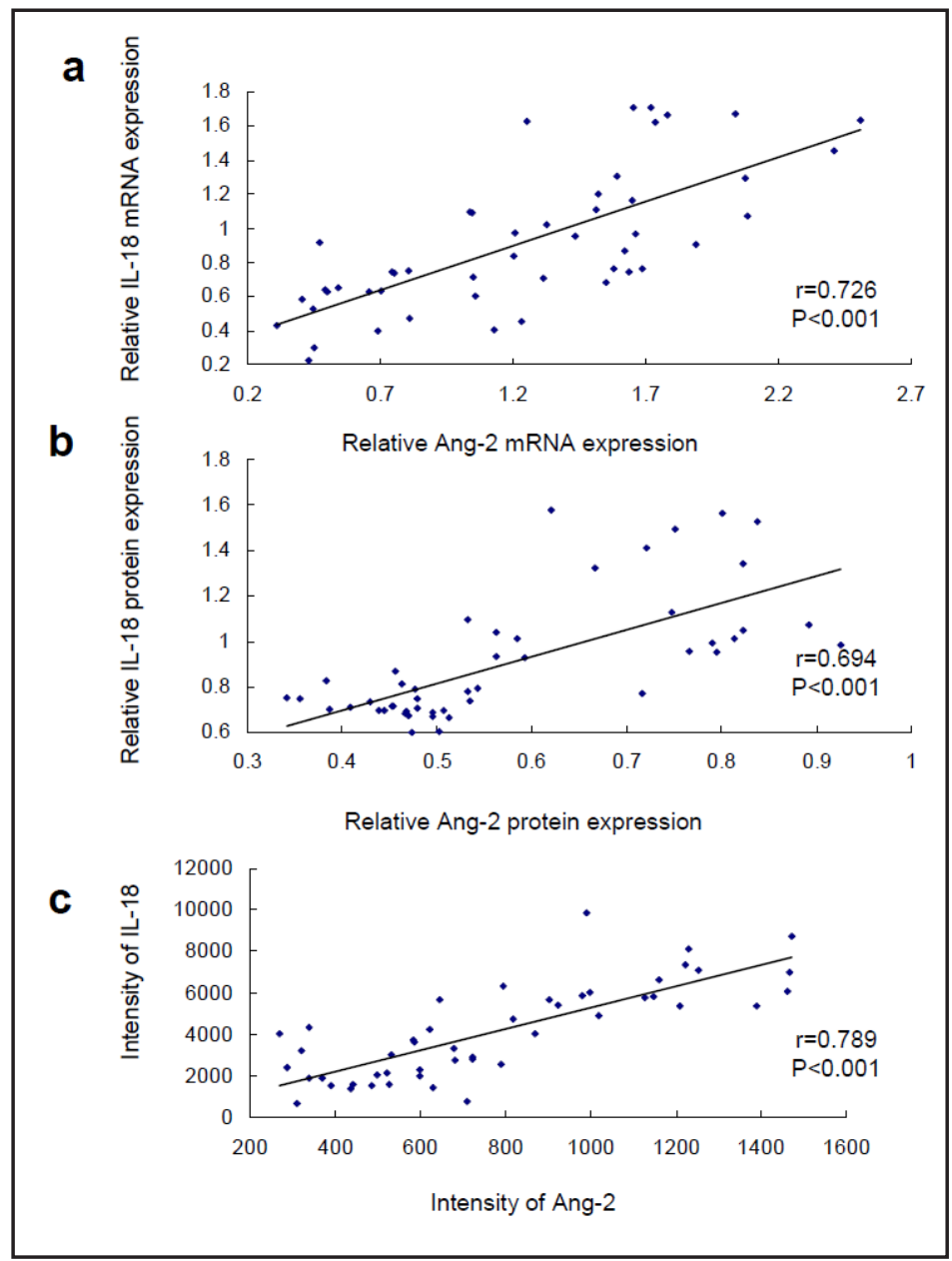

Alprostadil attenuated Ang-2 and IL-18 expression in cultured endothelial cells

Mouse endothelial cells were cultured with normal glucose or high glucose for 12, 24, 48 hours. Ang- 2 and IL-18 expression levels were significantly increased in endothelial cells by exposure to high glucose at 48 hours (Fig. 7a-d). Forty-eight hours of alprostadil treatment attenuated HG-induced Ang-2 and IL-18 expression, as shown in Fig. 7e-h. Incubation of cells with an osmotic control (30 mM of mannitol) did not reproduce the same effect (data not shown).

Ang-2 increased IL-18 expression in cultured endothelial cells under high glucose

To determine whether the levels of Ang-2 in GEnCs cells were sufficient to induce expression of IL-18, we measured levels of IL-18 protein. As shown in Fig.8, high levels of glucose could directly induce Ang-2 and IL-18 expression. Ang-2 had no effect on IL18 expression when GEnCs were incubated with $5 \mathrm{mM}$ glucose. However, Ang-2 further increased IL-18 protein levels in GEnC cells incubated with $30 \mathrm{mM}$ glucose. The effects of high glucose were partially rescued by treatment with Ang- 2 siRNA.

\section{Discussion}

DN is a common cause of end-stage kidney disease. Early symptoms of DN include increased glomerular and tubular epithelial cell hypertrophy and micro-albuminuria formation, thereby resulting in glomerular basement membrane thickening, mesangial matrix increase and proteinuria. The degree of proteinuria is closely related to glomerulosclerosis 


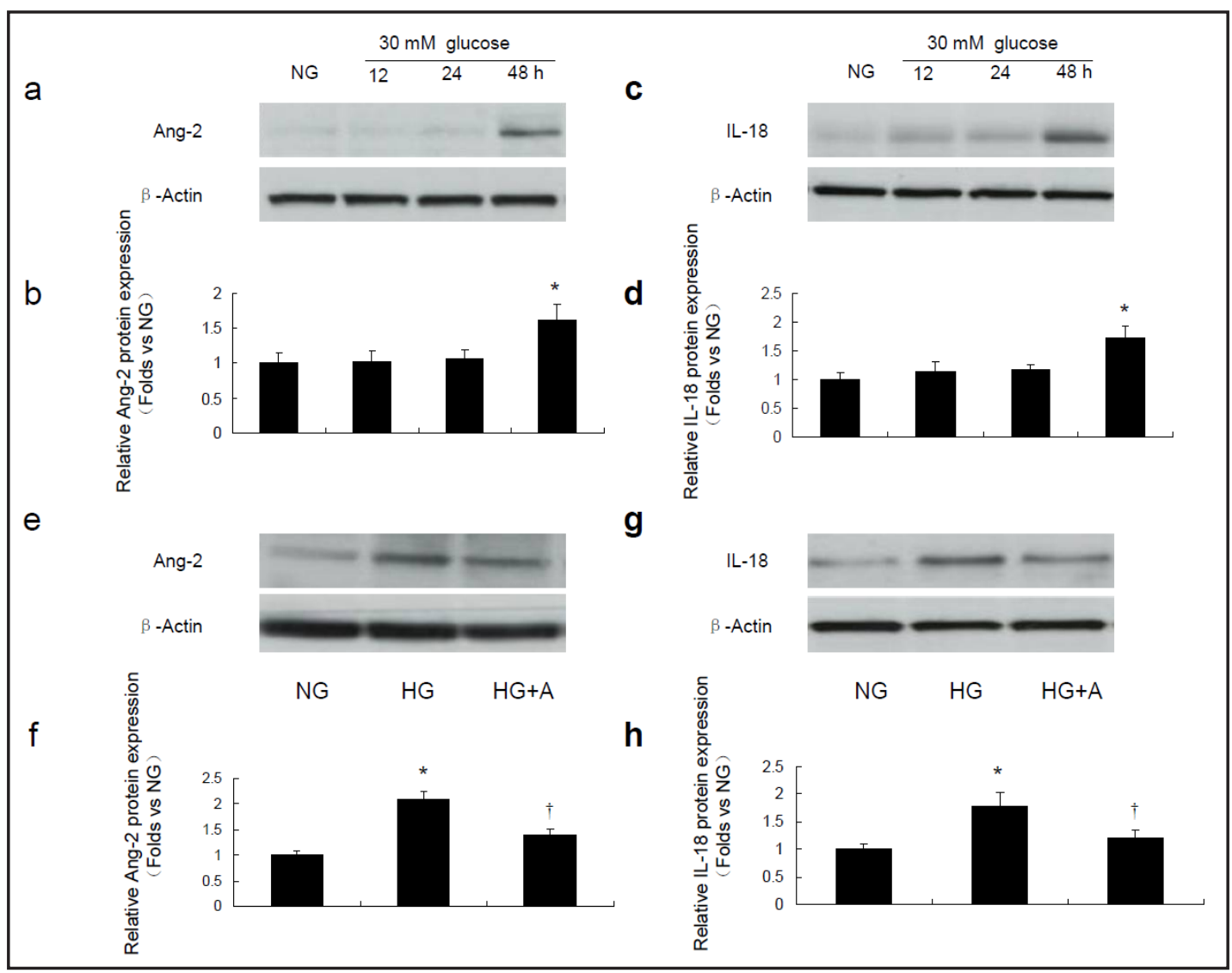

Fig. 7. Alprostadil attenuated Ang-2 and IL-18 protein expression in vitro. Western blot analysis of (a, b) Ang-2 and (c, d) IL-18 expression in cultured mouse glomerular endothelial cells under NG or HG for various time periods. Western blot analysis of (e, f) Ang-2 and (g, h) IL-18 protein levels in mouse glomerular endothelial cells treated with or without alprostadil for 48 hours. NG: normal glucose; HG: high glucose; A: alprostadil $* \mathrm{P}<0.05$ vs.NG, $+\mathrm{P}<0.05$ vs. HG. $(\mathrm{n}=4)$.

and tubule interstitial fibrosis progression [25], thus reducing urinary protein becomes critical in the treatment of DN.

Alprostadil (PGE1) is a widely used drug due to its effects of dilating blood vessels, inhibiting platelet aggregation and improving peripheral circulation. Clinical studies have shown that alprostadil can decrease proteinuria and protect renal function. Alprostadil was also reported to play a role in improving and protecting the kidneys through inhibition of the immune response, reducing kidney inflammation and reducing kidney cell apoptosis [1214]. However, the exact mechanisms remain unclear and is the focus of our current study.

Here we used a single intraperitoneal injection of STZ to induce DN in C57BL/6J mice and observed an increase in urinary protein, creatinine clearance rate, kidney/body weight ratio, glomerular volume and ECM. However, after alprostadil treatment, we show that urinary protein and creatinine clearance rate were significantly reduced along with attenuation of other pathological renal features, which is consistent with previous reports that alprostadil has a protective effect on the kidneys [12-14].

The mechanisms underlying the protective effect of alprostadil are unknown. Our attention drew to the angiopoietin family and inflammatory cytokines as potential regulators, since recent studies showed that Ang-2 is upregulated in DN and glomerulonephritis $[7,26]$. Ang-2 is stored in endothelial cell Weibel-Palade bodies and can be released by high-sugar, hypoxia, and inflammatory cytokines [27]. 
Fig. 8. Ang-2 can increase IL-18 expression under conditions of high glucose in vitro. Mouse glomerular endothelial cells were incubated in NG and $\mathrm{HG}$, alone or after transfection with Ang-2- pcDNA or Ang2 siRNA. Ang-2 and IL-18 protein levels were assessed by Western blot analysis (a) and the intensities relative to the $\beta$-actin (b). pcDNAAng-2 represents pcDNA3.1sigseq- myc-Ang2; siRNAAng-2 represents Ang-2 small interfering RNA. $* \mathrm{P}<0.05$ vs NG. $\dagger \mathrm{P}<0.05$ vs. HG. $(n=4)$.

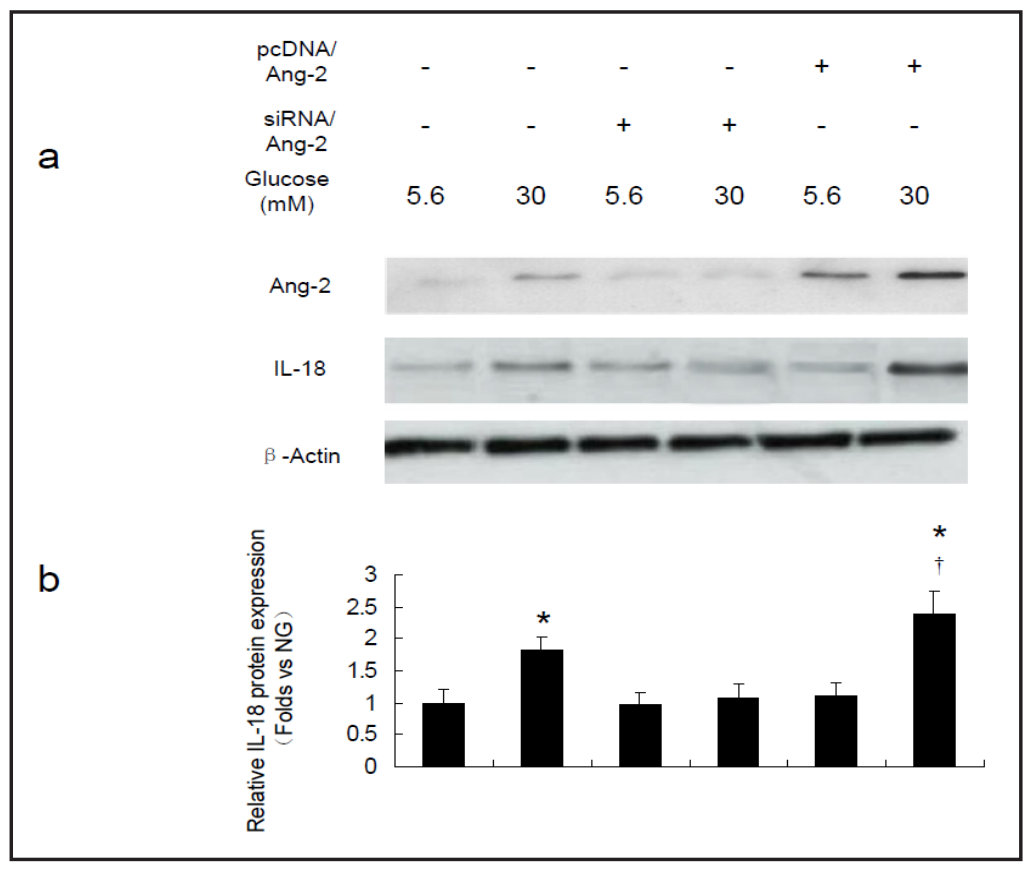

Research has also suggested that inflammation plays an important role in DN. For example, IL-18, a member of the IL-1 family, can enhance cytokine and chemokine generation [28]. Recent studies have shown that serum IL-18 level is increased in human acute hyperglycemia, as well as in patients with type 2 diabetes $[29,30]$. Natamura et al. also reported that blood and urine IL-18 levels are elevated in people with type 2 diabetes and DN [11].

In the present study, prolonged alprostadil treatment (more than 4 weeks) resulted in a significant reduction of both Ang-2 and IL-18 mRNA and protein levels in diabetic mice. In addition, high glucose-induced Ang- 2 and IL-18 expression was attenuated by alprostadil treatment in cultured glomerular endothelial cells. Both in vivo and in vitro results suggest that alprostadil might improve kidney function and protect mice from DN through regulation of these two genes.

Our data also revealed a previously unappreciated correlation between Ang-2 and IL-18. We found that both mRNA and protein levels of Ang-2 and IL-18 were positively correlated. In addition, Ang-2 mRNA was reduced in diabetic mice as short as two weeks after alprostadil treatment prior to alterations in Ang-2 protein levels and IL-18 gene expression, suggesting that the reno-protective effects of alprostadil are partly due to regulation of the Ang-2 gene at the early stages of DN. Ang-2 had no effect on IL-18 expression when GEnCs were incubated in glucose at normal levels. However, Ang-2 further increased IL-18 protein levels in GEnCs incubated in high glucose. The increased expression of Ang-2 in response to high glucose may lead to the increased expression of IL-18, These data provided evidence to suggest that high glucose-induced IL-18 production might be mediated by the induction of Ang-2 in glomerular endothelial cells, However, the mechanism needs further investigation.

In conclusion, our study provides new insights into the understanding of the molecular mechanisms underlying alprostadil protection on renal function. We also identified Ang-2 and IL-18 as potential therapeutic targets for DN treatment.

\section{Acknowledgement}

The study was supported by the National Natural Science Foundation of China (NSFC No. 30871174, No. 30800523, No. 81170662, No. 31200872, and No. 81300604) and the 
Luo et al.: Effect of Alprostadil in a Diabetic Nephropathy Model

Hubei Province Natural Science Fund of China (No. 2010CDB07906, No. 2011CHB014, No. 2012FFB02438, No. 2013 CFA026 and No. 2012 FFA038), and a Doctoral Fund of Ministry of Education of China (No. 20130142110064).

\section{Disclosure Statement}

All of the authors declared that there was no conflict of interest.

\section{References}

1 Maric-Bilkan C: Obesity and diabetic kidney disease. Med Clin North Am 2013;97:59-74.

- Hall PM: Prevention of Progression in Diabetic Nephropathy. Diabetes Spectr 2006;19:18-24.

3 Nakagawa T, Kosugi T, Haneda M, Rivard CJ, Long DA: Abnormal angiogenesis in diabetic nephropathy. Diabetes 2009;58:1471-1478.

4 Woolf AS, Gnudi L, Long DA: Roles of angiopoietins in kidney development and disease. J Am Soc Nephrol 2009;20:239-244.

5 Lim HS, Blann AD, Chong AY, Freestone B, Lip GY: Plasma vascular endothelial growth factor, angiopoietin-1, and angiopoietin-2 in diabetes: implications for cardiovascular risk and effects of multifactorial intervention. Diabetes Care 2004;27:2918-2924.

6 Yao D, Taguchi T, Matsumura T, Pestell R, Edelstein D, Giardino I, Suske G, Rabbani N, Thornalley PJ, Sarthy VP, Hammes HP, Brownlee M: High glucose increases angiopoietin-2 transcription in microvascular endothelial cells through methylglyoxal modification of mSin3A. J Biol Chem 2007;282:31038-31045.

7 Yamamoto Y, Maeshima Y, Kitayama H, Kitamura S, Takazawa Y, Sugiyama H, Yamasaki Y, Makino H: Tumstatin peptide, an inhibitor of angiogenesis, prevents glomerular hypertrophy in the early stage of diabetic nephropathy. Diabetes 2004;53:1831-1840.

8 Davis B, Dei Cas A, Long DA, White KE, Hayward A, Ku CH, Woolf AS, Bilous R, Viberti G, Gnudi L: Podocytespecific expression of angiopoietin-2 causes proteinuria and apoptosis of glomerular endothelia. J Am Soc Nephrol 2007;18:2320-2329.

9 Navarro-Gonzalez JF, Mora-Fernandez C: The role of inflammatory cytokines in diabetic nephropathy. J Am Soc Nephrol 2008;19:433-442.

10 Fujita T, Ogihara N, Kamura Y, Satomura A, Fuke Y, Shimizu C, Wada Y, Matsumoto K: Interleukin-18 contributes more closely to the progression of diabetic nephropathy than other diabetic complications. Acta Diabetol 2012;49:111-117.

11 Nakamura A, Shikata K, Hiramatsu M, Nakatou T, Kitamura T, Wada J, Itoshima T, Makino H: Serum interleukin-18 levels are associated with nephropathy and atherosclerosis in Japanese patients with type 2 diabetes. Diabetes Care 2005;28:2890-2895.

12 Li PF, Mu YR, Xin Y, Qu Y, Liao L: [Therapeutic effect of prostaglandin E1 on diabetic nephropathy: a oneyear follow-up study]. Nan Fang Yi Ke Da Xue Xue Bao 2010;30:482-485.

13 Miao Y, Zhong Y, Yan H, Li W, Wang BY, Jin J: Alprostadil plays a protective role in contrast-induced nephropathy in the elderly. Int Urol Nephrol 2013;45:1179-1185.

14 Wang H, Deng JL, Yue J, Li J, Hou YB: Prostaglandin E1 for preventing the progression of diabetic kidney disease. Cochrane Database Syst Rev 2010;10.1002/14651858.CD006872.pub2CD006872.

15 Feng Y, Wang Q, Wang Y, Yard B, Lang F: SGK1-mediated fibronectin formation in diabetic nephropathy. Cell Physiol Biochem 2005;16:237-244.

-16 Kosugi T, Yuzawa Y, Sato W, Kawai H, Matsuo S, Takei Y, Muramatsu T, Kadomatsu K: Growth factor midkine is involved in the pathogenesis of diabetic nephropathy. Am J Pathol 2006;168:9-19.

17 Wang Q, Zhang X, Wang Y, Deng A, Zhu Z, Feng Y: Significance and expression of serum and glucocorticoidinducible kinase in kidney of mice with diabetic nephropathy. J Huazhong Univ Sci Technolog Med Sci 2005;25:170-173. 
Luo et al.: Effect of Alprostadil in a Diabetic Nephropathy Model

18 Yang RH, Wang F, Hou XH, Cao ZP, Wang B, Xu XN, Hu SJ: Dietary omega-3 polyunsaturated fatty acids improves learning performance of diabetic rats by regulating the neuron excitability. Neuroscience 2012;212:93-103.

19 Luo C, Zhang X, Wang Y, Zhu Z, Deng A: Effect of lipo-prostaglandin E1 on mesangial proliferative glomerulonephritis in rats. J Huazhong Univ Sci Technolog Med Sci 2005;25:516-518.

-20 Lu YH, Deng AG, Li N, Song MN, Yang X, Liu JS: Changes in angiopoietin expression in glomeruli involved in glomerulosclerosis in rats with daunorubicin-induced nephrosis. Acta Pharmacol Sin 2006;27:579-587.

-21 Hagele H, Allam R, Pawar RD, Reichel CA, Krombach F, Anders HJ: Double-stranded DNA activates glomerular endothelial cells and enhances albumin permeability via a toll-like receptor-independent cytosolic DNA recognition pathway. Am J Pathol 2009;175:1896-1904.

-22 Rops AL, van der Vlag J, Jacobs CW, Dijkman HB, Lensen JF, Wijnhoven TJ, van den Heuvel LP, van Kuppevelt $\mathrm{TH}$, Berden JH: Isolation and characterization of conditionally immortalized mouse glomerular endothelial cell lines. Kidney Int 2004;66:2193-2201.

-23 Wang Y, Landheer S, van Gilst WH, van Amerongen A, Hammes HP, Henning RH, Deelman LE, Buikema H: Attenuation of renovascular damage in Zucker diabetic fatty rat by NWT-03, an egg protein hydrolysate with ACE- and DPP4-inhibitory Activity. PLoS One 2012;7:e46781.

24 Yan Q, Sui W, Wang B, Zou H, Zou G, Luo H: Expression of MMP-2 and TIMP-1 in renal tissue of patients with chronic active antibody-mediated renal graft rejection. Diagn Pathol 2012;7:141.

25 Wolf G, Ziyadeh FN: Cellular and molecular mechanisms of proteinuria in diabetic nephropathy. Nephron Physiol 2007;106:p26-31.

-26 Ichinose K, Maeshima Y, Yamamoto Y, Kitayama H, Takazawa Y, Hirokoshi K, Sugiyama H, Yamasaki Y, Eguchi K, Makino H: Antiangiogenic endostatin peptide ameliorates renal alterations in the early stage of a type 1 diabetic nephropathy model. Diabetes 2005;54:2891-2903.

-27 Fiedler U, Scharpfenecker M, Koidl S, Hegen A, Grunow V, Schmidt JM, Kriz W, Thurston G, Augustin HG: The Tie-2 ligand angiopoietin-2 is stored in and rapidly released upon stimulation from endothelial cell WeibelPalade bodies. Blood 2004;103:4150-4156.

28 Lebel-Binay S, Berger A, Zinzindohoue F, Cugnenc P, Thiounn N, Fridman WH, Pages F: Interleukin-18: biological properties and clinical implications. Eur Cytokine Netw 2000;11:15-26.

-29 Araki S, Haneda M, Koya D, Sugimoto T, Isshiki K, Chin-Kanasaki M, Uzu T, Kashiwagi A: Predictive impact of elevated serum level of IL-18 for early renal dysfunction in type 2 diabetes: an observational follow-up study. Diabetologia 2007;50:867-873.

-30 Esposito K, Nappo F, Marfella R, Giugliano G, Giugliano F, Ciotola M, Quagliaro L, Ceriello A, Giugliano D: Inflammatory cytokine concentrations are acutely increased by hyperglycemia in humans: role of oxidative stress. Circulation 2002;106:2067-2072. 